\title{
Training and Practices of Cannabis Dispensary Staff
}

Nancy A. Haug, ${ }^{1,2, *}$ Dustin Kieschnick, ${ }^{1}$ James E. Sottile, ${ }^{3}$ Kimberly A. Babson, ${ }^{4}$

Ryan Vandrey, ${ }^{5}$ and Marcel O. Bonn-Miller ${ }^{4,6-8}$

\begin{abstract}
Introduction: The proliferation of cannabis dispensaries within the United States has emerged from patient demand for the legalization of cannabis as an alternative treatment for a number of conditions and symptoms. Unfortunately, nothing is known about the practices of dispensary staff with respect to recommendation of cannabis strains/concentrations for specific patient ailments. To address this limitation, the present study assessed the training and practices of cannabis dispensary staff.

Materials and Methods: Medical and nonmedical dispensary staff $(n=55)$ were recruited via e-mail and social media to complete an online survey assessing their demographic characteristics, dispensary features, patient characteristics, formal training, and cannabis recommendation practices.

Results: Fifty-five percent of dispensary staff reported some formal training for their position, with $20 \%$ reporting medical/scientific training. A majority (94\%) indicated that they provide specific cannabis advice to patients. In terms of strains, dispensary staff trended toward recommendations of Indica for anxiety, chronic pain, insomnia, nightmares, and Tourette's syndrome. They were more likely to recommend Indica and hybrid plants for posttraumatic stress disorder (PTSD)/trauma and muscle spasms. In contrast, staff were less likely to recommend Indica for depression; hybrid strains were most often recommended for amyotrophic lateral sclerosis (ALS). In terms of cannabinoid concentrations, dispensary staff were most likely to recommend a 1:1 ratio of delta-9-tetrahydrocannabinol (THC):cannabidiol (CBD) for patients suffering from anxiety, Crohn's disease, hepatitis C, and PTSD/trauma, while patients seeking appetite stimulation were most likely to be recommended THC. Staff recommended high CBD for arthritis and Alzheimer's disease and a high CBD or 1:1 ratio for ALS, epilepsy, and muscle spasms.

Conclusions: Although many dispensary staff are making recommendations consistent with current evidence, some are recommending cannabis that has either not been shown effective for, or could exacerbate, a patient's condition. Findings underscore the importance of consistent, evidence-based, training of dispensary staff who provide specific recommendations for patient medical conditions.
\end{abstract}

Keywords: cannabis; marijuana; dispensary; practices

\section{Introduction}

Approximately half of the United States has legalized cannabis for medicinal purposes, with four states having also legalized cannabis for nonmedical use. Epidemiological research suggests that despite its association with negative short- and long-term effects such as addiction, deficits in cognitive performance and motor coordination, and psychosis, ${ }^{1}$ a number of particularly vulnerable groups of individuals are using cannabis to alleviate their medical conditions (e.g., anxiety, chronic

${ }^{1}$ PGSP-Stanford Psy.D. Consortium, Palo Alto University, Palo Alto, California.

${ }^{2}$ Department of Psychiatry and Behavioral Sciences, Stanford University School of Medicine, Stanford, California.

${ }^{3}$ Pacific Graduate School of Psychology, Palo Alto University, Palo Alto, California.

${ }^{4}$ National Center for PTSD, VA Palo Alto Healthcare System, Palo Alto, California.

${ }^{5}$ Department of Psychiatry and Behavioral Sciences, Johns Hopkins University School of Medicine, Baltimore, Maryland.

${ }^{6}$ Center for Innovation to Implementation, VA Palo Alto Healthcare System, Palo Alto, California.

${ }^{7}$ Center of Excellence in Substance Abuse Treatment and Education, Philadelphia VAMC, Philadelphia, Pennsylvania.

${ }^{8}$ Department of Psychiatry, University of Pennsylvania Perelman School of Medicine, Philadelphia, Pennsylvania.

*Address correspondence to: Nancy A. Haug, PhD, Palo Alto University, 5150 El Camino Real, C-24, Los Altos, CA 94022, E-mail: nhaug@paloaltou.edu

(C) Nancy A. Haug et al. 2016; Published by Mary Ann Liebert, Inc. This Open Access article is distributed under the terms of the Creative Commons License (http://creativecommons.org/licenses/by/4.0), which permits unrestricted use, distribution, and reproduction in any medium, provided the original work is properly credited. 
pain, epilepsy, cancer, HIV/AIDS, post-traumatic stress disorder [PTSD]). ${ }^{2,3}$ Indeed, more than 1,000 medical cannabis dispensaries, cooperatives, and delivery services are operating in California, ${ }^{4}$ and $\sim 500$ exist in Colorado, to meet patient demand.

Although each state has created its own legislation to govern the cultivation and distribution of cannabis to individuals, there is currently little to no guidance or oversight of associated patient care. Indeed, with the exception of a few states that have mandated cannabisspecific physician continuing medical education (e.g., New York), the majority of states do not require any training for either those providing "recommendations" for patient cannabis use (i.e., physicians) or those actually dispensing cannabis to consumers (i.e., dispensaries and/or "bud tenders"). This is troubling, as cannabis comprises more than 400 chemical compounds and is associated with widely variable effects among humans. ${ }^{5}$ To provide a specific example, empirical literature has shown that delta-9-tetrahydrocannabinol (THC), the primary psychoactive compound in the cannabis plant, can be anxiogenic, while cannabidiol (CBD), a secondary cannabinoid, has anxiolytic effects. ${ }^{6}$ Next, the literature would suggest that the provision of cannabis, comprising high levels of THC, to individuals with anxiety may be contraindicated.

Although it is important to note that rigorous research on the use of cannabis as a therapeutic remains in its relative infancy, issues of inconsistent and nonempirically supported practices by physicians plague the cannabis and substance use field more broadly. ${ }^{7}$ So, to offer initial information regarding current practices by those providing cannabis recommendations to patients, the present study aimed to document the training and practices of a sample of dispensary staff (i.e., "bud tenders"). Given its descriptive nature, no specific hypotheses were forwarded; however, information garnered from this study is meant to inform targeted implementation science efforts aimed at streamlining provider practices and highlighting the need for structured education in this emerging industry that has developed outside traditional mechanisms of medical drug development.

\section{Materials and Methods \\ Participants}

Participants included 55 self-identified dispensary staff members who provided informed consent to complete an online survey. Dispensary types included medical (59\%), nonmedical (18\%), and both medical and non- medical (23\%). The dispensaries were located in Colorado (41\%), California (20\%), Arizona (16\%), Oregon (2\%), District of Columbia (5\%), and the Northeast (10\%; Connecticut, Rhode Island, Massachusetts, Maine). The locations of the dispensaries were self-reported as follows: rural (16\%), suburban (13\%), small city $(<300,000$; $35 \%)$, and large city $(>300,000 ; 36 \%)$.

\section{Measures}

An online survey was constructed by the study investigators to evaluate the training, knowledge, attitudes, and practices of dispensary staff. Questions included demographics (i.e., age, race/ethnicity, sexual orientation, marital status, education, annual income, dispensary earnings, and hours worked), dispensary features (i.e., geographical location, zip code, type of dispensary), and a checklist of primary responsibilities. A dichotomous (yes/no) item assessed formal dispensary training, and if endorsed, the item branched to a checklist of types of training (e.g., medical, scientific, business, customer service) with a textbox for "other." Additional items assessed the number of patients served, the percentage of patients who are repeat patrons, and a categorical response choice for how often repeat customers visit the dispensary. The typical amount of cannabis purchased per visit was assessed by having the participant fill in an amount in grams, ounces, or dollars. A dichotomous (yes/no) item assessed whether advice, guidance, or counsel is provided to patients, and if endorsed, the item branched to a checklist of types of advice (e.g., benefits of cannabis and side effects) with a textbox for "other." Items assessing the medical/psychological symptoms or conditions reported by patients were indexed on a 3-point Likert scale ranging from "rarely" to "frequently." Respondents also checked off which cannabinoid concentrations (i.e., high THC, high CBD, 1:1 ratio of $\mathrm{THC} / \mathrm{CBD}$ ) or cannabis plant strain recommendations (i.e., sativa, indica, hybrid) they made for each of the symptoms or conditions listed. Attitudes toward work (e.g., satisfaction, feeling valued, stigma, burnout) were assessed on a 5-point Likert scale ranging from "very satisfied" to "very dissatisfied" or "always" to "never."

\section{Procedure}

The study was approved by the Palo Alto University Institutional Review Board (FWA00010885; Protocol\# 15-001-S). A Federal Certificate of Confidentiality was obtained from the National Institutes of Health to protect participant confidentiality. Dispensaries were identified 
via website finders (i.e., leafly.com, weedmaps.com) and a contact list from the Americans for Safe Access. From September 2015 through May 2016, dispensary staff were invited via e-mail ( $n=550$, with $10 \%$ returned as undeliverable and $20 \%$ providing an automated e-mail response) and/or telephone $(n=117)$ to complete an anonymous survey. A direct link to the survey was also posted on a cannabis advocacy organization Facebook page (i.e., National Organization for the Reform of Marijuana Laws) and a Reddit subreddit geared toward dispensary staff. Of those who accessed the survey or clicked on the survey link, $87 \%$ provided informed consent to continue. On completion of the survey, 33 participants were placed in a lottery drawing for a $\$ 25$ Amazon.com gift card. To increase rates of participation in a later wave of recruitment, each participant was compensated with a $\$ 10$ Amazon.com gift card $(n=22)$.

Survey data were analyzed cross-sectionally using IBM SPSS Statistics, version 23. Descriptive statistics provided a profile of dispensary staff characteristics. One-sample $t$-tests for proportions were calculated to compare cannabinoid and strain recommendations for each patient symptom or condition. Since the survey allowed for participants to skip items, the reported numbers for each item may differ.

\section{Results}

\section{Participant characteristics}

The mean age of participants was 31.9 years (standard deviation $[\mathrm{SD}]=9.8$ years), with a range of $22-63$ years. Majority were Caucasian (86\%) and 9\% identified as having Hispanic or Latino ethnicity. The sexual orientation of participants was heterosexual (67\%), bisexual (22\%), homosexual (7\%), and asexual (4\%). The sample was $55 \%$ female, $33 \%$ reported being married or partnered, and $60 \%$ had a college degree or higher. Most (84\%) reported working at the dispensary full-time $(>30 \mathrm{~h})$ at an average of $\$ 15.00 / \mathrm{h} \quad(\mathrm{SD}=\$ 4.60 ; \quad$ Range $=\$ 8.00$ $\$ 25.00 / \mathrm{h}$ ). The average duration of employment at the current dispensary ranged from 1 month to 7 years with a median of 1 year ( $M=21.6$ months, $S D=20.0$ months). Twenty percent of the sample reported working at another dispensary before their current position.

\section{Dispensary staff training and responsibilities}

Dispensary staff were asked whether they received any formal training for their current position and the type of training received. In our sample, $55 \%(n=30)$ of staff members reported some formal training. The types of training included customer service $(35 \% ; n=19)$, busi- ness $(26 \% ; n=14)$, medical $(20 \% ; n=11)$, other $(20 \%$; $n=11)$, and scientific $(13 \% ; n=7)$. Other training consisted of "bud tender" certification, or courses on cannabis (e.g., Cannabis 101), or safety and regulatory compliance (e.g., SellSmart, METRC).

The dispensary staff described their primary job responsibilities as follows: customer service $(91 \% ; n=39)$, stocking inventory $(79 \% ; n=34)$, ordering supplies or dealing with vendors/growers $(67 \% ; n=29)$, counseling patients $(63 \% ; n=27)$, record-keeping $(63 \% ; n=27)$, budgeting/finances/accounting (46\%; $n=20)$, and other responsibilities $(25 \% ; n=14)$ such as human resources, delivery, marketing, packaging products, and creating signage.

\section{Dispensary patients}

The number of patients served by the dispensaries ranged from 15 to 5,000 patients per week, with a median of 425 patients $(M=778.4, S D=1001.5, n=50)$. On average, $\mathrm{M}=69 \%(\mathrm{SD}=21 \%)$ of patients were described as repeat or frequent patrons. Repeat patrons visited the dispensary daily $(26 \% ; n=13), 2$ to 3 times per week $(40 \% ; n=20)$, once a week $(22 \% ; n=11)$, or 2 to 3 times per month $(12 \% ; n=6)$. The average amount of cannabis purchased per visit was reported as $\mathrm{M}=10.4 \mathrm{~g} \quad(\mathrm{SD}=9.4 \mathrm{~g}, \quad n=31)$ or $\mathrm{M}=\$ 83.00$ $(\mathrm{SD}=\$ 32.00$, Range $=\$ 25.00-\$ 150.00, n=18)$.

Dispensary staff members were queried on the symptoms or conditions frequently reported by their patients (see Fig. 1 for a summary). The most frequent symptoms included chronic pain $(93 \% ; n=41)$, insomnia $(80 \% ; n=35)$, and anxiety $(80 \% ; n=35)$. Approximately two-thirds of the sample $(62 \% ; n=26)$ reported that they always or often check in or follow-up about their patients' health status.

\section{Dispensary staff recommendations}

Dispensary staff were queried regarding the specific recommendations they make to patients and on what information those recommendations are based. A majority $(94 \% ; n=47)$ reported that they provide advice, guidance, or counsel to patients. The type of advice included information on particular cannabis strains (88\%; $n=44)$, suggested administration methods (88\%; $n=44)$, potential cannabis side effects $(80 \% ; n=40)$, benefits of cannabis for specific symptoms $(74 \% ; n=37)$, and other recommendations $(22 \% ; n=11)$ such as natural remedies, travel/shipping legal advice, dosing guidelines, and ailment or disease-specific information. Those who did not provide advice or counsel indicated that it was 


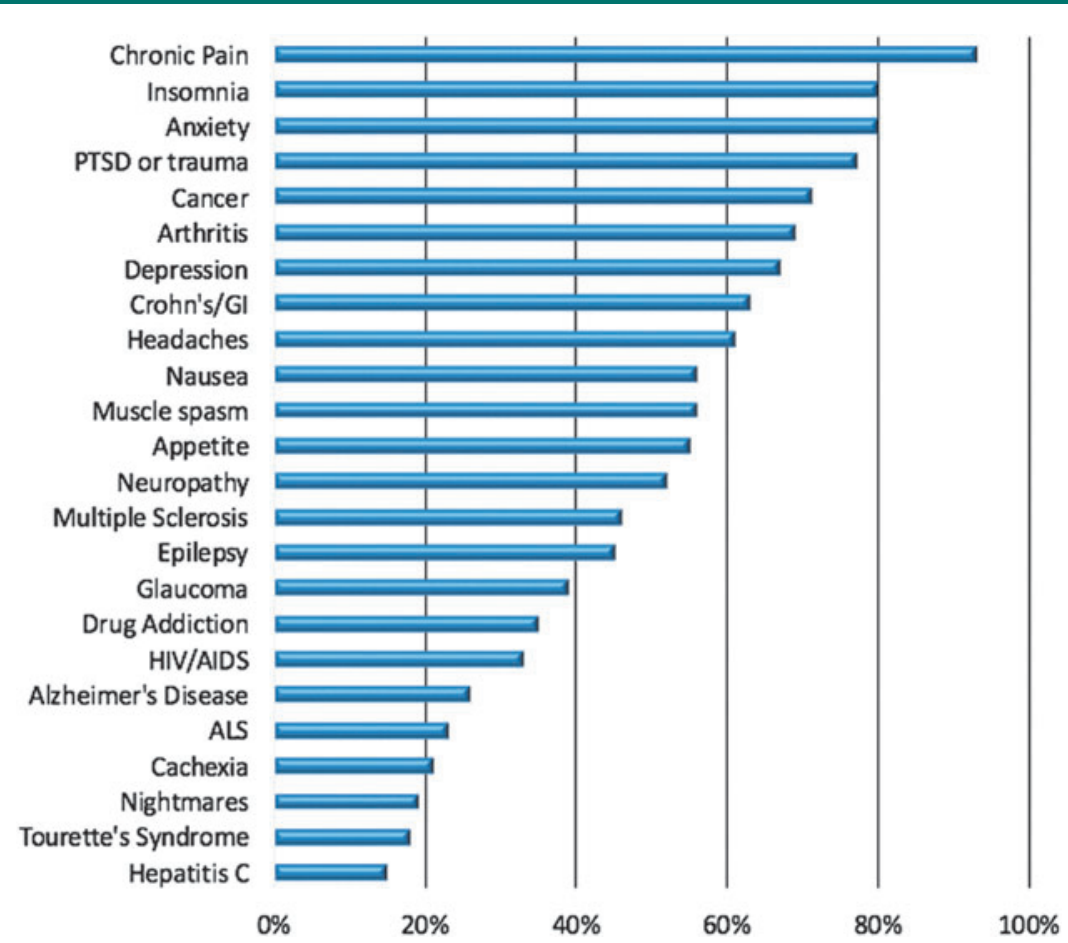

FIG. 1. The percentage of dispensary staff who reported frequently seeing patients with the following symptoms or conditions. ALS, amyotrophic lateral sclerosis; Gl, gastrointestinal; PTSD, post-traumatic stress disorder.

not part of their role at the dispensary (i.e., driver or delivery service). None of the dispensary staff members indicated that they provide advice regarding medications or drugs other than cannabis to their patients.

Dispensary staff reported making recommendations for specific strains or formulas of cannabis based on the following: the particular condition or ailment $(89 \%$; $n=39)$, the experience of other patients $(83 \% ; n=35)$, patient preference or needs $(79 \% ; n=33)$, their own experience $(71 \% ; n=30)$, information obtained from scientific articles $(68 \% ; n=28)$, dispensary owner or other staff recommendations (52\%; $n=22)$, information obtained on websites $(48 \% ; n=20)$, new variety or unusual/rare breed $(47 \% ; n=20)$, and what needs to get moved out of inventory (21\%; $n=9)$.

The survey assessed which plant strains (i.e., sativa, indica, hybrid) and which cannabinoid concentrations (i.e., high THC, high CBD, 1:1 ratio of THC/CBD) were recommended for particular symptoms or conditions. Several patient conditions were associated with specific plant strain recommendations by dispensary staff (Table 1). Indeed, dispensary staff were more likely to recommend indica for chronic pain and Tourette's syndrome than sativa. They were also more likely to recommend indica for insomnia, anxiety, and nightmares than both sativa and hybrid plants. They were more likely to recommend indica and hybrid plants for PTSD or trauma, and muscle spasms compared to sativa. In contrast, staff were less likely to recommend indica for depression than sativa or hybrid plants. Finally, dispensary staff recommended hybrid strains more often for ALS than sativa.

In terms of specific cannabinoid recommendations, dispensary staff were more likely to recommend a 1:1 ratio of THC:CBD for anxiety, PTSD or trauma, and Crohn's disease compared to high THC. They were more likely to recommend high $\mathrm{CBD}$ and a 1:1 ratio for ALS, epilepsy, and muscle spasms compared to high THC. Dispensary staff were more likely to recommend high CBD than high THC for arthritis and Alzheimer's disease. They were also more likely to recommend a 1:1 ratio for hepatitis $\mathrm{C}$ compared to high THC or high CBD. Staff were more likely to recommend high THC for appetite than high CBD (Table 2).

\section{Dispensary staff attitudes}

Dispensary staff members were asked to rate satisfaction with their current position on a scale ranging 
Table 1. Dispensary Staff Plant Strain Recommendations for Patient Symptoms or Conditions ${ }^{\mathrm{a}}$

\begin{tabular}{|c|c|c|c|c|}
\hline Symptom or condition & $S, \%$ & $\mathrm{I}, \%$ & $H, \%$ & Significance \\
\hline ALS or Lou Gehrig's disease & 11 & 21 & 36 & $t(27)=2.07, p=0.048(\mathrm{~S}$ vs. H) \\
\hline Alzheimer's disease & 14 & 18 & 18 & ns \\
\hline Anxiety & 13 & 60 & 23 & $\begin{array}{l}t(29)=3.61, p=0.001 \text { (S vs. I) } \\
t(29)=2.43, p=0.021(\text { I vs. H) }\end{array}$ \\
\hline Appetite & 53 & 33 & 33 & ns \\
\hline Arthritis & 25 & 36 & 32 & ns \\
\hline Cachexia (wasting syndrome) & 32 & 36 & 32 & ns \\
\hline Cancer & 36 & 36 & 46 & ns \\
\hline Chronic pain & 23 & 57 & 30 & $t(29)=2.25, p=0.032($ S vs. I) \\
\hline Crohn's disease/GI & 21 & 43 & 25 & ns \\
\hline Depression & 63 & 16 & 47 & $\begin{array}{l}t(29)=3.41, p=0.002(\mathrm{~S} \text { vs. I) } \\
t(29)=2.32, p=0.027(\text { I vs. H) }\end{array}$ \\
\hline Drug addiction & 27 & 36 & 39 & ns \\
\hline Epilepsy & 14 & 18 & 18 & ns \\
\hline Glaucoma & 32 & 25 & 32 & ns \\
\hline Headaches or migraines & 27 & 23 & 37 & ns \\
\hline Hepatitis C & 18 & 21 & 25 & ns \\
\hline HIV/AIDS & 27 & 36 & 32 & ns \\
\hline Insomnia & 3 & 80 & 20 & $\begin{array}{l}t(29)=8.66, p=0.000(\mathrm{~S} \text { vs. I) } \\
t(29)=4.11, p=0.000(\mathrm{I} \text { vs. } \mathrm{H})\end{array}$ \\
\hline Multiple sclerosis & 18 & 29 & 36 & ns \\
\hline Muscle spasms & 7 & 40 & 33 & $\begin{array}{l}t(29)=3.01, p=0.005(\mathrm{~S} \text { vs. I) } \\
t(29)=2.47, p=0.020(\mathrm{~S} \text { vs. H) }\end{array}$ \\
\hline Nausea & 27 & 33 & 47 & ns \\
\hline Neuropathy & 17 & 43 & 40 & ns \\
\hline Nightmares & 13 & 70 & 20 & $\begin{array}{l}t(29)=4.39, p=0.000(\mathrm{~S} \text { vs. I) } \\
t(29)=3.40, p=0.002(\mathrm{I} \text { vs. } \mathrm{H})\end{array}$ \\
\hline PTSD or trauma & 11 & 46 & 36 & $\begin{array}{l}t(27)=2.77, p=0.010(\mathrm{~S} \text { vs. I) } \\
t(27)=2.07, p=0.058(\mathrm{~S} \text { vs. H) }\end{array}$ \\
\hline Tourette's syndrome & 11 & 39 & 25 & $t(27)=2.28, p=0.031$ (S vs. I) \\
\hline
\end{tabular}

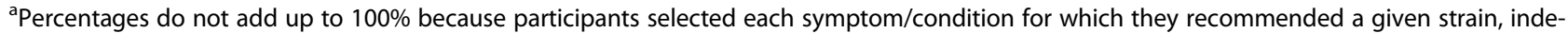
pendently. Thus, symptoms/conditions for which percentages added up to above $100 \%$ indicate that some participants recommended multiple strains for a given condition. Conversely, symptoms/conditions for which percentages added up to less than 100\% indicate that some participants did not recommend any strain for a given condition.

$\mathrm{Gl}$, gastrointestinal; $\mathrm{H}$, hybrid; I, indica; ns, not significant; PTSD, post-traumatic stress disorder; S, sativa.

from very dissatisfied (1) to very satisfied (5). The mean score for satisfaction was $4.27(\mathrm{SD}=0.95, n=41)$, indicating a high level of satisfaction with their work.

Other attitudes toward dispensary work were assessed on a scale ranging from never (1) to always (5). Items included the following: feeling valued or appreciated because of work $(\mathrm{M}=3.81, \mathrm{SD}=1.13, n=42)$, feeling stigmatized or looked down upon by others for their work $(\mathrm{M}=2.57 ; \mathrm{SD}=1.06, n=42)$, and experiencing burnout or fatigue as a result of work $(M=2.83$, $\mathrm{SD}=1.10, n=42$ ).

\section{Discussion}

While a number of studies have examined the characteristics of patients seeking cannabis recommendations from a physician, ${ }^{3,8}$ and those obtaining cannabis from a dispensary, ${ }^{2,9}$ the present study serves as the first examination of characteristics and practices of dispensary staff. Findings indicate that the vast majority of staff provide specific counseling to patients regarding cannabis that may be most helpful for their individual con- ditions, and that these recommendations are based on a number of sources, both empirically and nonempirically based. Despite the vast number of staff members offering recommendations to patients, only $20 \%$ of our sample reported prior medical and/or scientific training.

In terms of plant strain recommendations, very little work has documented patient preference or the specific efficacy of certain cannabis strains as a function of clinical condition. Staff recommendation of indica strains for chronic pain in the current study was consistent with individual patient preference observed in other work. ${ }^{10}$ The recommendation of indica primarily for insomnia and nightmares is consistent with one patient survey, ${ }^{11}$ but differs from another empirical study that highlighted patient preference for sativa strains for sleep difficulties. ${ }^{12}$ While observations related to strain recommendations are interesting, due to extensive hybridization and variations in growing conditions, the differences between cannabis strains do not seem to play as large a role in determining subjective effects as cannabinoid concentrations. ${ }^{13}$ This has led some to 
Table 2. Dispensary Staff Cannabinoid Recommendations for Patient Symptoms or Conditions ${ }^{\mathrm{a}}$

\begin{tabular}{|c|c|c|c|c|}
\hline Symptom or condition & $\begin{array}{l}\text { High THC } \\
\text { (THC), \% }\end{array}$ & $\begin{array}{l}\text { High CBD } \\
\text { (CBD), \% }\end{array}$ & $\begin{array}{c}\text { 1:1 Ratio } \\
\text { THC/CBD }(1: 1), \%\end{array}$ & Significance \\
\hline ALS or Lou Gehrig's disease & 18 & 57 & 57 & $t(27)=2.67, p=0.013($ THC vs. CBD, THC vs. $1: 1)$ \\
\hline Alzheimer's disease & 21 & 61 & 50 & $t(27)=2.61, p=0.015(\mathrm{THC}$ vs. CBD) \\
\hline Anxiety & 13 & 30 & 40 & $t(29)=2.19, p=0.037(\mathrm{THC}$ vs. $1: 1)$ \\
\hline Appetite & 63 & 10 & 7 & $\begin{array}{l}t(29)=4.93, p=0.000(\text { THC vs. } 1: 1) \\
t(29)=4.33, p=0.000(\text { THC vs. CBD) }\end{array}$ \\
\hline Arthritis & 32 & 71 & 53 & $t(27)=2.20, p=0.036(\mathrm{THC}$ vs. CBD) \\
\hline Cachexia (wasting syndrome) & 54 & 32 & 43 & ns \\
\hline Cancer & 50 & 57 & 68 & ns \\
\hline Chronic pain & 33 & 53 & 57 & ns \\
\hline Crohn's disease/Gl & 25 & 47 & 61 & $t(27)=2.23, p=0.034($ THC vs. $1: 1)$ \\
\hline Depression & 33 & 26 & 53 & ns \\
\hline Drug addiction & 36 & 42 & 50 & ns \\
\hline Epilepsy & 7 & 61 & 54 & $\begin{array}{l}t(27)=4.59, p=0.000(\text { THC vs. CBD) } \\
t(27)=3.99, p=0.000(\text { THC vs. } 1: 1)\end{array}$ \\
\hline Glaucoma & 39 & 36 & 50 & ns \\
\hline Headaches or migraines & 33 & 37 & 63 & ns \\
\hline Hepatitis C & 18 & 29 & 64 & $\begin{array}{l}t(27)=3.12, p=0.004(\text { THC vs. } 1: 1) \\
t(27)=2.07, p=0.049(\text { CBD vs. } 1: 1)\end{array}$ \\
\hline HIV/AIDS & 36 & 36 & 68 & ns \\
\hline Insomnia & 33 & 33 & 40 & ns \\
\hline Multiple sclerosis & 32 & 46 & 57 & ns \\
\hline Muscle spasms & 10 & 53 & 53 & $t(29)=3.53, p=0.001($ THC vs. CBD, THC vs. 1:1) \\
\hline Nausea & 50 & 23 & 53 & ns \\
\hline Neuropathy & 20 & 47 & 47 & ns \\
\hline Nightmares & 20 & 43 & 37 & ns \\
\hline PTSD or trauma & 21 & 28 & 57 & $t(27)=2.36, p=0.026($ THC vs. $1: 1)$ \\
\hline Tourette's syndrome & 18 & 43 & 43 & ns \\
\hline
\end{tabular}

aPercentages do not add up to $100 \%$ because participants selected each symptom/condition for which they recommended a given cannabinoid concentration, independently. Thus, symptoms/conditions for which percentages added up to above $100 \%$ indicate that some participants recommended multiple cannabinoid concentrations for a given condition. Conversely, symptoms/conditions for which percentages added up to less than $100 \%$ indicate that some participants did not recommend any cannabinoid concentration for a given condition.

$\mathrm{CBD}$, cannabidiol; THC, tetrahydrocannabinol.

argue that distinctions among cannabis chemovars labeled as "Sativa" or "Indica" are relatively meaningless unless accompanied by detailed accurate assays of cannabinoid and terpenoid content. ${ }^{14}$

Dispensary staff recommendations of cannabinoid concentrations (e.g., THC, CBD) for particular patient symptoms and conditions were also frequently incongruent with the existing empirical literature. For instance, the existing empirical literature would suggest that cannabis high in CBD could be particularly helpful for individuals with anxiety disorders, while cannabis high in THC could actually lead to acute and longterm anxiety reactions. ${ }^{6,15,16}$ Early pre-clinical work has also highlighted CBD as a potential antidepressant, ${ }^{15}$ anticonvulsant, ${ }^{16,17}$ and therapeutic for PTSD. ${ }^{15}$ On the other end of the spectrum, the literature would suggest that THC may be an antiemetic, and particularly beneficial for appetite stimulation and pain. ${ }^{18}$ Finally, a number of reviews have documented that a combination of THC and CBD (e.g., nabiximols) can have therapeutic value for individuals with spasticity due to multiple sclerosis, ${ }^{16,19}$ neuro- pathic pain, ${ }^{16,20}$ as well as sleep disturbances. ${ }^{15}$ Based on the above literature, it appears that a meaningful number of dispensary staff are providing recommendations for cannabinoids that have either not been shown to be effective for a given condition (e.g., 33\% recommending THC for depression, $10 \%$ recommending CBD for appetite, $78 \%$ recommending either high THC or high CBD for multiple sclerosis), or could actually worsen a patient's condition (e.g., 13\% recommending THC for anxiety, 7\% recommending THC for epilepsy).

Aside from the present study's contribution to the literature in terms of describing the characteristics of cannabis dispensary staff, findings highlight the importance of consistent, evidence-based training of those providing specific recommendations of cannabis strains or cannabinoid concentrations for a given patient condition (e.g., physicians, dispensary staff). Indeed, while one might expect the most qualified individuals to provide specific recommendations of cannabis product to be trained physicians who are aware of a patient's medical history and other prescription medications that could interact with certain cannabinoids, ${ }^{21-23}$ it is dispensary 
staff who are the most likely to provide cannabis advice. As each state is currently responsible for drafting and monitoring its own cannabis legislation, it is imperative for states to mandate some form of educational certification for any individual providing cannabis advice to patients, not just physicians. Furthermore, given the quickly evolving literature in this field, it seems necessary for individuals to receive regular updates via continuing medical education. Given the increasing availability and preferences for myriad methods of cannabis consumption (e.g., edibles, extracts, dabs) ${ }^{2,24}$ and corresponding risks, ${ }^{25,26}$ patient and provider education programs should strive to include trainings in safe and effective use of cannabis as a function of preparation.

The present study data, although novel, are not without limitations. First, given the cross-sectional nature of the investigation, we were unable to determine changes in staff behavior over time or as a function of the implementation of particular training programs. Second, while the utilization of structured surveys allowed for the systematic and quantitative analysis of staff characteristics and practices, such surveys are limited in that they do not allow for nuanced information regarding staff behavior. Future prospective qualitative work that incorporates open-ended questions would aid in fleshing out the present findings. Third, although both medicinal and nonmedical dispensary staff were recruited, higher response rates in specific states may limit generalizability of findings to dispensaries across the United States or in other countries. Web-based recruitment methods also limit our ability to determine overall sample representativeness. Indeed, the survey response rate may have been restricted by perceived stigma associated with both medical and nonmedical cannabis use and/or skepticism of researchers by dispensary staff. Finally, the current study aimed to describe overall behavior among dispensary staff, and in doing so the survey did not collect information about physician practices or detailed information about the provision of services for certain, particularly vulnerable, populations (e.g., individuals with PTSD). Limitations notwithstanding, the present study serves as a "call to action" for consistent and evidence-based training of providers in the cannabis industry.

\section{Acknowledgments}

The authors thank Steph Sherer, Executive Director of Americans for Safe Access (ASA), and Dr. Mitch Earleywine, member of the Board of Directors for the National Organization for the Reform of Marijuana
Laws (NORML), for their assistance in the recruitment of dispensary staff.

\section{Author Disclosure Statement}

Drs. Bonn-Miller and Vandrey serve as consultants for CW Botanicals, Insys Therapeutics, and Zynerba Pharmaceuticals. Dr. Bonn-Miller also consults for Tilray (a division of Privateer Holdings). Dr. Babson served as a consultant for Insys Therapeutics. None of the above entities was involved with the design, implementation, analysis, or dissemination of the present study. The expressed views do not necessarily represent those of the Department of Veterans Affairs.

\section{References}

1. Volkow ND, Baler RD, Compton WM, et al. Adverse health effects of marijuana use. N Engl J Med. 2014;370:2219-2227.

2. Bonn-Miller MO, Boden MT, Bucossi MM, et al. Self-reported cannabis use characteristics, patterns and helpfulness among medical cannabis users. Am J Drug Alcohol Abuse. 2014;40:23-30.

3. Nunberg $\mathrm{H}$, Kilmer B, Pacula RL, et al. An analysis of applicants presenting to a medical marijuana specialty practice in California. J Drug Policy Anal. 2011;4:1-14.

4. Reinarman $\mathrm{C}$, Nunberg $\mathrm{H}$, Lanthier $\mathrm{F}$, et al. Who are medical marijuana patients? Population characteristics from nine California assessment clinics. J Psychoactive Drugs. 2011;43:128-135.

5. Russo EB. Taming THC: potential cannabis synergy and phytocannabinoid-terpenoid entourage effects. Br J Pharmacol. 2011;163:1344-1364.

6. Crippa JA, Zuardi AW, Martin-Santos R, et al. Cannabis and anxiety: a critical review of the evidence. Hum Psychopharmacol. 2009;24: 515-523.

7. Bujarski SJ, Galang JN, Short NA, et al. Cannabis use disorder treatment barriers and facilitators among veterans with PTSD. Psychol Addict Behav. 2016;30:73-81.

8. Ilgen MA, Bohnert K, Kleinberg F, et al. Characteristics of adults seeking medical marijuana certification. Drug Alcohol Depend. 2013;132:654-659.

9. Zaller N, Topletz A, Frater S, et al. Profiles of medicinal cannabis patients attending compassion centers in Rhode Island. J Psychoactive Drugs. 2015;47:18-23.

10. Cohen NL, Heinz AJ, Ilgen $M$, et al. Pain, Cannabis species, and Cannabis use disorders. J Stud Alcohol Drugs. 2016;77:515-520.

11. Pearce DD, Mitsouras K, Irizarry KJ. Discriminating the effects of Cannabis sativa and Cannabis indica: a web survey of medical cannabis users. J Altern Complement Med. 2014;20:787-791.

12. Belendiuk KA, Babson KA, Vandrey R, et al. Cannabis species and cannabinoid concentration preference among sleep-disturbed medicinal cannabis users. Addict Behav. 2015;50:178-181.

13. Small E. Evolution and classification of cannabis sativa (marijuana, hemp) in relation to human utilization. Bot Rev. 2015;81:189-294.

14. Piomelli D, Russo EB. The Cannabis sativa versus Cannabis indica debate: an interview with Ethan Russo, MD. Cannabis Cannabinoid Res. 2016;1:44-46.

15. Loflin MJ, Babson KA, Bonn-Miller MO. Cannabinoids as therapeutic for PTSD: a review. Curr Opin Psychol. 2016; in press.

16. Morabito $D$, Soyster $P$, Ramey-Wright $S$, et al. A review of recent advances in the therapeutic uses of secondary cannabinoids. Curr Addict Rep. 2016;3:230-238.

17. Rosenberg EC, Tsien RW, Whalley BJ, et al. Cannabinoids and epilepsy. Neurotherapeutics. 2015;12:747-768.

18. Whiting PF, Wolff RF, Deshpande S, et al. Cannabinoids for medical use: a systematic review and meta-analysis. JAMA. 2015;313:2456-2473.

19. Syed YY, McKeage K, Scott LJ. Delta-9-tetrahydrocannabinol/cannabidiol (Sativex $(R))$ : a review of its use in patients with moderate to severe spasticity due to multiple sclerosis. Drugs. 2014;74:563-578. 
20. Snedecor SJ, Sudharshan L, Cappelleri JC, et al. Systematic review and meta-analysis of pharmacological therapies for painful diabetic peripheral neuropathy. Pain Pract. 2014;14:167-184.

21. Shafaroodi H, Samini M, Moezi L, et al. The interaction of cannabinoids and opioids on pentylenetetrazole-induced seizure threshold in mice. Neuropharmacology. 2004;47:390-400.

22. Kumar S, Rao PS, Earla R, et al. Drug-drug interactions between antiretroviral therapies and drugs of abuse in HIV systems. Expert Opin Drug Metab Toxicol. 2015;11:343-355.

23. Friedman D, Cilio MR, Tilton $\mathrm{N}$, et al. The effect of Epidiolex (Cannabidiol) on serum levels of concomitant anti-epileptic drugs in children and young adults with treatment-resistant epilepsy in an expanded access program. American Epilepsy Society: Seattle, WA, 2014.

24. Bonn-Miller MO, Sottile JE, Padula CB, et al. Routes of cannabis consumption as a function of clinical status. College on Problems of Drug Dependence: Palm Springs, CA, 2016.

25. Loflin $M$, Earleywine $M$. A new method of cannabis ingestion: the dangers of dabs? Addict Behav. 2014;39:1430-1433.

26. Vandrey R, Raber JC, Raber ME, et al. Cannabinoid dose and label accuracy in edible medical cannabis products. JAMA. 2015;313:2491-2493.
Cite this article as: Haug NA, Kieschnick D, Sottile JE, Babson KA, Vandrey R, Bonn-Miller MO (2016) Training and practices of cannabis dispensary staff, Cannabis and Cannabinoid Research 1:1, 244-251, DOI: 10.1089/can.2016.0024.

\section{Publish in Cannabis and Cannabinoid Research}

Cannabis and

Cannabinoid

Research
- Immediate, unrestricted online access

- Rigorous peer review

- Compliance with open access mandates

- Authors retain copyright

- Highly indexed

- Targeted email marketing 